\title{
EQUIDISTRIBUTION OF RATIONAL FUNCTIONS HAVING A SUPERATTRACTING PERIODIC POINT TOWARDS THE ACTIVITY CURRENT AND THE BIFURCATION CURRENT
}

\author{
YÛSUKE OKUYAMA
}

\begin{abstract}
We establish an approximation of the activity current $T_{c}$ in the parameter space of a holomorphic family $f$ of rational functions having a marked critical point $c$ by parameters for which $c$ is periodic under $f$, i.e., is a superattracting periodic point. This partly generalizes a Dujardin-Favre theorem for rational functions having preperiodic points, and refines a Bassanelli-Berteloot theorem on a similar approximation of the bifurcation current $T_{f}$ of the holomorphic family $f$. The proof is based on a dynamical counterpart of this approximation.
\end{abstract}

\section{INTRODUCTION}

The $J$-stable locus $S_{f}$ in a holomorphic family $f$ of rational functions is open and dense in the parameter space, contains the quasiconformally stable locus of $f$ as an open and dense subset, and is characterized by the non-activity of all the critical points if they are marked [21] (see also [22, Chapter 4] and [20]). The J-unstable locus or the bifurcation locus $B_{f}$ of $f$ can also be studied from a pluripotential theoretical viewpoint. Our aim is to contribute to the study of the instability in a holomorphic family of rational functions and the activity of its marked critical point. We give an affirmative answer, in the superattracting case, to a question on the removability of a seemingly technical assumption on the parameter space posed by Dujardin-Favre [14, Theorem 4.2], and refines a result due to Bassanelli-Berteloot [3, Theorem $3.1(1)$ ]. See also survey articles [6] and [13].

1.1. Equidistribution towards the activity current $T_{c}$. We say a mapping $f: \Lambda \times \mathbb{P}^{1} \rightarrow \mathbb{P}^{1}$ is a holomorphic family of rational functions on $\mathbb{P}^{1}$ of degree $d>1$ over a connected complex manifold $\Lambda$ if $f$ is holomorphic and for every $\lambda \in \Lambda$, $f_{\lambda}:=f(\lambda, \cdot)$ is a rational function on $\mathbb{P}^{1}$ of degree $d$, and say that $f$ has a marked critical point $c: \Lambda \rightarrow \mathbb{P}^{1}$ if $c$ is holomorphic and for every $\lambda \in \Lambda, c(\lambda)$ is a critical point of $f_{\lambda}$. I].

For the details of pluripotential theory, we refer to [10, Chapter III] and [18, Part

Definition 1.1. Let $\phi, \psi$ be meromorphic functions on a connected complex manifold $M$. If $\phi \not \equiv \psi$ on $M$, then let $[\phi=\psi]$ be the current of integration over the divisor defined by the equation $\phi=\psi$ on $M$ : the Poincaré-Lelong formula asserts

Received by the editors February 24, 2014 and, in revised form, July 11, 2014 and August 12, 2014.

2010 Mathematics Subject Classification. Primary 37F45.

Key words and phrases. Holomorphic family, marked critical point, superattracting periodic point, equidistribution, activity current, bifurcation current. 
that when $\phi$ and $\psi$ are holomorphic, $\mathrm{dd}^{c} \log |\phi-\psi|=[\phi=\psi]$ (for $\mathrm{dd}^{c}$, see Notation 2.1). On the other hand, by convention, if $\phi \equiv \psi$, then we set $[\phi=\psi]:=0$ as a $(1,1)$-current on $M$; we should be careful for this convention since in the case that $\phi \equiv 0$ and $\psi \neq \equiv 0$ on $M,[\phi=0]+[\psi=0]=[\psi=0]$ might be not equal to $[(\phi \psi)=0]=0$.

For each $n \in \mathbb{N}$, set

$$
F_{n}(\lambda):=f_{\lambda}^{n}(c(\lambda)) \quad \text { on } \Lambda .
$$

Definition 1.2 (The currents $\operatorname{Per}_{c}(n)$ and $\operatorname{Per}_{c}^{*}(n)$ ). Following [14, Definition 4.1], for every $n \in \mathbb{N}$, set

$$
\operatorname{Per}_{c}(n):=\left[F_{n}=c\right] \text { on } \Lambda \text {. }
$$

Moreover, for each $n \in \mathbb{N}$, let $X_{n}$ be the closure in $\Lambda$ of

$$
\operatorname{supp}\left[F_{n}=c\right] \backslash\left(\bigcup_{m \in \mathbb{N}: m \mid n \text { and } m<n} \operatorname{supp}\left[F_{m}=c\right]\right),
$$

which is also an analytic subset in $\Lambda$ and whose irreducible components are those of $\operatorname{supp} \operatorname{Per}_{c}(n)$. Denoting by $[A]$ the current of integration over an analytic variety $A$ in $\Lambda$, we set

$$
\operatorname{Per}_{c}^{*}(n):=\sum_{V}\left(\operatorname{ord}_{V}\left(\operatorname{Per}_{c}(n)\right)\right) \cdot[V],
$$

where the sum ranges over all irreducible components $V$ of $X_{n}$.

Definition 1.3. Let $\omega$ be the Fubini-Study area element on $\mathbb{P}^{1}$ normalized as $\omega\left(\mathbb{P}^{1}\right)=1$. To the marked critical point $c$ of $f$, we can associate the activity current

$$
T_{c}:=\lim _{n \rightarrow \infty} \frac{F_{n}^{*} \omega}{d^{n}} \quad \text { as a }(1,1) \text {-current on } \Lambda .
$$

The proof of the convergence of the right hand side is due to [14, PropositionDefinition 3.1] (see also Remark 2.3). The support of $T_{c}$ coincides with the activity locus

$$
A_{c}:=\left\{\lambda \in \Lambda:\left\{F_{n}: n \in \mathbb{N}\right\} \text { is not normal at } \lambda\right\}
$$

associated to $c([14$, Theorem 3.2]).

The following is our principal result: the convergence (1.4) partially generalizes Dujardin-Favre [14, Theorem 4.2] by removing their technical assumption in our superattracting case. The foundational case that $f(\lambda, z)=z^{d}+\lambda$ and $c \equiv 0$ on $\Lambda=\mathbb{C}$ was due to Levin [19].

Theorem 1. Let $f: \Lambda \times \mathbb{P}^{1} \rightarrow \mathbb{P}^{1}$ be a holomorphic family of rational functions on $\mathbb{P}^{1}$ of degree $d>1$ over a connected complex manifold $\Lambda$ having a marked critical point $c: \Lambda \rightarrow \mathbb{P}^{1}$. Then

$$
\begin{gathered}
\lim _{n \rightarrow \infty} \frac{\operatorname{Per}_{c}(n)}{d^{n}+1}=T_{c} \quad \text { as currents on } \Lambda, \quad \text { and } \\
\lim _{n \rightarrow \infty} \frac{\operatorname{Per}_{c}^{*}(n)}{d^{n}+1}=T_{c} \quad \text { as currents on } \Lambda .
\end{gathered}
$$


Remark 1.4. Both (1.4) and (1.5) hold even if $F_{n} \equiv c$ on $\Lambda$ for some $n \in \mathbb{N}$ : for, in this case, $\#\left\{F_{n}: n \in \mathbb{N}\right\}<\infty$, so $\lim _{n \rightarrow \infty} \operatorname{Per}_{c}(n) /\left(d^{n}+1\right)=\lim _{n \rightarrow \infty} \operatorname{Per}_{c}^{*}(n) /\left(d^{n}+\right.$ 1) $=0$ as currents on $\Lambda$ and also $A_{c}=\emptyset$. The latter implies $T_{c}=0$ on $\Lambda$ since $\operatorname{supp} T_{c} \subset A_{c}$.

Our proof of Theorem 1 relies on a dynamical counterpart of this result, and is simpler than the Dujardin-Favre argument, which relies on a delicate classification [14, Theorem 4] of non-active parameters.

1.2. Equidistribution towards the bifurcation current $T_{f}$. Let $f: \Lambda \times \mathbb{P}^{1} \rightarrow$ $\mathbb{P}^{1}$ be a holomorphic family of rational functions of degree $d>1$ over a connected complex manifold $\Lambda$.

For every $\lambda \in \Lambda$, let $L\left(f_{\lambda}\right)$ be the Lyapunov exponent of $f_{\lambda}$ with respect to the unique maximal entropy measure of $f_{\lambda}$. The function $\Lambda \ni \lambda \mapsto L\left(f_{\lambda}\right) \in \mathbb{R}$ is positive, continuous, and plurisubharmonic on $\Lambda$.

Definition 1.5 (DeMarco [11, Theorem 1.1]; see also Pham [26] and Dinh-Sibony $[12, \S 2.5])$. The bifurcation current $T_{f}$ on $\Lambda$ of $f$ is defined by

$$
T_{f}:=\operatorname{dd}_{\lambda}^{c} L\left(f_{\lambda}\right) \quad \text { as a }(1,1) \text {-current on } \Lambda .
$$

Taking a finitely-sheeted possibly ramified covering of $\Lambda$ if necessary, we can assume that there are marked critical points $c_{1}, \ldots, c_{2 d-2}: \Lambda \rightarrow \mathbb{P}^{1}$ of $f$ such that for every $\lambda \in \Lambda, c_{1}(\lambda), \ldots, c_{2 d-2}(\lambda)$ are all the critical points of $f_{\lambda}$ taking into account their multiplicities. Then by DeMarco's formula [11, Theorem 1.4] (see also Remark 2.3), $T_{f}$ is decomposed as

$$
T_{f}=\sum_{j=1}^{2 d-2} T_{c_{j}} .
$$

Definition 1.6 (A periodic point having the exact period). Fix $n \in \mathbb{N}$ and $\lambda \in \Lambda$. A fixed point $w \in \mathbb{P}^{1}$ of $f_{\lambda}^{n}$ is a periodic point of $f_{\lambda}$ having the exact period $n$ if for every $m \in \mathbb{N}$ satisfying $m \mid n$ and $m<n, f_{\lambda}^{m}(w) \neq w$. Let $\operatorname{Fix}^{*}\left(f_{\lambda}^{n}\right)$ be the set of all periodic points of $f_{\lambda}$ having the exact period $n$.

For each $n \in \mathbb{N}$, the holomorphic family $f$ induces the multiplier polynomial $p_{n}^{*}(\lambda, w)=p_{f, n}^{*}(\lambda, w)$ on $\Lambda \times \mathbb{C}$, which satisfies that $(\lambda, w) \mapsto p_{n}^{*}(\lambda, w)$ is a holomorphic function on $\Lambda \times \mathbb{C}$, that for each $\lambda \in \Lambda, p_{n}^{*}(\lambda, \cdot)$ is a polynomial on $\mathbb{C}$, and that for every $w \in \mathbb{C} \backslash\{1\}$ (the description when $w=1$ is a little complicated) and every $\lambda \in \Lambda, p_{n}^{*}(\lambda, w)=0$ if and only if there exists $z_{0} \in \operatorname{Fix}^{*}\left(f_{\lambda}^{n}\right)$ satisfying $\left(f_{\lambda}^{n}\right)^{\prime}\left(z_{0}\right)=w\left(p_{n}^{*}\right.$ was introduced by Morton-Vivaldi [24, $\S 1$ ] working on integral domains $R$ more general than $\mathbb{C})$. For the precise definition of $p_{n}^{*}(\lambda, w)$, see Definition 4.8, in the case $w=0$, for every $n \in \mathbb{N}$ and every $\lambda \in \Lambda$,

$$
\left|p_{n}^{*}(\lambda, 0)\right|=\prod_{z \in \operatorname{Fix}^{*}\left(f_{\lambda}^{n}\right)}\left|f_{\lambda}^{\prime}(z)\right| .
$$

Following 44, §2], for each $w \in \mathbb{C}$, set

$$
\operatorname{Per}_{f}^{*}(n, w):=\left[p_{n}^{*}(\cdot, w)=0\right] \quad \text { on } \Lambda
$$

(the suffix $*$ is added to the original notation $\operatorname{Per}_{f}(n, w)$ in [4, §2]).

The convergence (1.5) is regarded as a refinement of the following. 
Theorem 1.7 (Bassanelli-Berteloot [4, Theorem 3.1 (1)]). Let $f: \Lambda \times \mathbb{P}^{1} \rightarrow \mathbb{P}^{1}$ be a holomorphic family of rational functions on $\mathbb{P}^{1}$ of degree $d>1$ over a connected complex manifold $\Lambda$. Then

$$
\lim _{n \rightarrow \infty} \frac{\operatorname{Per}_{f}^{*}(n, 0)}{d^{n}}=T_{f} \quad \text { as currents on } \Lambda .
$$

See Section 6 for the deduction of Theorem 1.7 from Theorem 1 This proof of Theorem 1.7 is simpler than the proof of Bassanelli and Berteloot, which relies on an approximation formula of the Lyapunov exponent of a rational function by the multipliers of its repelling periodic points.

Remark 1.8. The full statement of Bassanelli-Berteloot [4, Theorem 3.1] can be deduced from Theorem 1.7 (see also Bassanelli-Berteloot [3, §3]). For further studies, see also Buff-Gauthier [9] and Gauthier [15].

1.3. Organization of this article. In Section 2 we recall a reduction (1.4l) of (1.4) in Theorem 1 as in [14, Proof of Theorem 4.2], and in Section 3, we show a dynamical counterpart of (1.4]). In Section 4. we recall a local description of $\operatorname{Per}_{c}^{*}(n)$, a global decomposition of $\operatorname{Per}_{c}(n)$, and the definition of $p_{n}^{*}(\lambda, w)$. In Section 5, we show Theorem 1 based on this dynamical counterpart (plowing in the dynamical space and reaping in the parameter space; see, e.g., [8, §1.1]). In Section 6] we establish a local decomposition of $\operatorname{Per}_{f}^{*}(n, 0)$ and show Theorem 1.7 using Theorem 1 .

\section{A reduction of Theorem 1}

Notation 2.1. As in Section 1 let $\omega$ be the normalized Fubini-Study area element on $\mathbb{P}^{1}$. Let $\|\cdot\|$ be the Euclidean norm on $\mathbb{C}^{2}$. The origin of $\mathbb{C}^{2}$ is also denoted by 0 , and $\pi: \mathbb{C}^{2} \backslash\{0\} \rightarrow \mathbb{P}^{1}$ is the canonical projection. Setting the wedge product $\left(z_{0}, z_{1}\right) \wedge\left(w_{0}, w_{1}\right):=z_{0} w_{1}-z_{1} w_{0}$ on $\mathbb{C}^{2} \times \mathbb{C}^{2}$, the normalized chordal metric $[z, w]$ on $\mathbb{P}^{1}$ is the function

$$
(z, w) \mapsto[z, w]:=|p \wedge q| /(\|p\| \cdot\|q\|)(\leq 1)
$$

on $\mathbb{P}^{1} \times \mathbb{P}^{1}$, where $p \in \pi^{-1}(z), q \in \pi^{-1}(w)$. We normalize $\mathrm{dd}^{c}$ as $\mathrm{d}=\partial+\bar{\partial}$ and $\mathrm{d}^{c}=i(\bar{\partial}-\partial) /(2 \pi)$. Then $\pi^{*} \omega=\mathrm{dd}^{c} \log \|\cdot\|$ as currents on $\mathbb{C}^{2} \backslash\{0\}$.

Let $f: \Lambda \times \mathbb{P}^{1} \rightarrow \mathbb{P}^{1}$ be a holomorphic family of rational functions on $\mathbb{P}^{1}$ of degree $d>1$ over a connected complex manifold $\Lambda$ having a marked critical point $c: \Lambda \rightarrow \mathbb{P}^{1}$. Recall that $F_{n}(\lambda):=f_{\lambda}^{n}(c(\lambda))$ on $\Lambda$ for each $n \in \mathbb{N}$.

The following reduction of (1.4) in Theorem 1 is due to Dujardin-Favre.

Lemma 2.2 ([14, in Proof of Theorem 4.2]). Let $f, c$, and $F_{n}$ be as in the above. Then the convergence (1.4) in Theorem 1 holds if

$$
\lim _{n \rightarrow \infty} \frac{\log \left[F_{n}, c\right]}{d^{n}+1}=0 \quad \text { in } L_{\mathrm{loc}}^{1}(\Lambda) .
$$

Let us prove Lemma 2.2. For every point $\lambda_{0} \in \Lambda$ and every open and connected neighborhood $U$ of $\lambda_{0}$ in $\Lambda$ small enough, there is a lift $\tilde{c}: U \rightarrow \mathbb{C}^{2} \backslash\{0\}$ of $c$ in that $\tilde{c}$ is holomorphic and that $\pi \circ \tilde{c}=c$ on $U$, and there is a lift $\tilde{f}: U \times \mathbb{C}^{2} \rightarrow \mathbb{C}^{2}$ of $f$ in that $\tilde{f}$ is holomorphic and that for every $\lambda \in U, \tilde{f}_{\lambda}:=\tilde{f}(\lambda, \cdot)$ is a homogeneous 
polynomial endomorphism on $\mathbb{C}^{2}$ satisfying $\pi \circ \tilde{f}_{\lambda}=f_{\lambda} \circ \pi$ on $\mathbb{C}^{2} \backslash\{0\}$ and $\tilde{f}_{\lambda}^{-1}(0)=$ $\{0\}$. For each $n \in \mathbb{N}$, set

$$
\tilde{F}_{n}(\lambda):=\tilde{f}_{\lambda}^{n}(\tilde{c}(\lambda)) \quad \text { on } U \text {. }
$$

Recall the definition of $T_{c}$ (Definition 1.3) and that $F_{n}^{*} \omega=\operatorname{dd}^{c} \log \left\|\tilde{F}_{n}\right\|$ as currents on $U$. In particular,

$$
\lim _{n \rightarrow \infty} \operatorname{dd}^{c} \frac{\log \left\|\tilde{F}_{n}\right\|}{d^{n}}=T_{c} \quad \text { as currents on } U
$$

(see, e.g., [6, Lemma 3.2.7]). By Remark 1.4, we can assume that $F_{n} \not \equiv c$ on $\Lambda$ for every $n \in \mathbb{N}$. Then for every $n \in \mathbb{N}$, by the Poincaré-Lelong formula,

$$
\operatorname{dd}^{c} \log \left|\tilde{F}_{n} \wedge \tilde{c}\right|=\left[\tilde{F}_{n} \wedge \tilde{c}=0\right]=\left[F_{n}=c\right]=: \operatorname{Per}_{c}(n)
$$

as currents on $U$. By (2.1), for every $n \in \mathbb{N}$,

$$
\log \left|\tilde{F}_{n} \wedge \tilde{c}\right|=\log \left[F_{n}, c\right]+\log \left\|\tilde{F}_{n}\right\|+\log \|\tilde{c}\| \quad \text { on } U,
$$

so that the continuity of $\mathrm{dd}^{c}$ on $L_{\text {loc }}^{1}(\Lambda)$ completes the proof of Lemma 2.2.

We also recall that the dynamical Green function of $\tilde{f}$ is the local uniform limit

$$
G^{\lambda}(p):=\lim _{n \rightarrow \infty} \frac{\log \left\|\tilde{f}_{\lambda}^{n}(p)\right\|}{d^{n}}
$$

on $U \times\left(\mathbb{C}^{2} \backslash\{0\}\right)$ (see, e.g., [2, Proposition 1.2]). In particular,

$$
\lim _{n \rightarrow \infty} \frac{\log \left\|\tilde{F}_{n}(\lambda)\right\|}{d^{n}}=G^{\lambda}(\tilde{c}(\lambda)) \quad \text { locally uniformly on } U \text {. }
$$

Remark 2.3. The locally uniform convergence (2.6) implies not only (1.3) but also $T_{c}=\operatorname{dd}_{\lambda}^{c} G^{\lambda}(\tilde{c}(\lambda))$ on $U$, which with DeMarco's formula $L\left(f_{\lambda}\right)=-\log d+$ $\sum_{j=1}^{2 d-2} G^{\lambda}\left(\tilde{c}_{j}(\lambda)\right)-(2 / d) \log \left|\operatorname{Res}\left(\tilde{f}_{\lambda}\right)\right|$, where $\operatorname{Res}\left(\tilde{f}_{\lambda}\right)$ is the homogeneous resultant of $\tilde{f}_{\lambda}$, on $U$ implies (1.6).

\section{A dynamical counterpart of (1.4]}

For the details of complex dynamics, see, e.g., 23].

Definition 3.1. Let $f$ be a rational function on $\mathbb{P}^{1}$. The Julia set of $f$ is defined by $J(f):=\left\{z \in \mathbb{P}^{1}:\left\{f^{n}: n \in \mathbb{N}\right\}\right.$ is not normal at $\left.z\right\}$, whose complement in $\mathbb{P}^{1}$ is called the Fatou set of $f$ and denoted by $F(f)$.

The following is a dynamical counterpart of (1.4]).

Lemma 3.2. Let $f$ be a rational function on $\mathbb{P}^{1}$ of degree $d>1$. If a critical point $c$ of $f$ is not periodic under $f$, then

$$
\lim _{n \rightarrow \infty} \frac{\log \left[f^{n}(c), c\right]}{d^{n}+1}=0 .
$$

Proof. Suppose that $c \in F(f)$ and contrary that (3.1) does not hold, i.e.,

$$
\liminf _{n \rightarrow \infty} \frac{\log \left[f^{n}(c), c\right]}{d^{n}+1}<0 .
$$

Then the Fatou component $U$ containing $c$ must intersect $f^{n}(U)$ for some $n \in \mathbb{N}$, so that $U$ is a cyclic Fatou component of $f$ having, say, the period $m \in \mathbb{N}$. By the local non-injectivity of $f$ at $c, f^{m}: U \rightarrow U$ is not univalent. Then by the Denjoy-Wolff theorem (and the hyperbolicity of $U$, cf. [23, $\S 5$ and $\S 16]$ ), (3.2) even implies that 
$c$ is a (super)attracting periodic point of $f$, which contradicts the non-periodicity assumption on $c$ under $f$. Hence (3.1) holds in this case.

Suppose next that $c \in J(f)$. Then (3.1) follows from (the proof of) Przytycki [27. Lemma 1], which asserts that for every critical point $c \in J(f)$ of $f$ and every $n \in \mathbb{N},\left[f^{n}(c), c\right] \geq 1 /\left(20 L^{n}\right)$, where $L>1$ is a Lipschitz constant of $f: \mathbb{P}^{1} \rightarrow \mathbb{P}^{1}$ with respect to the normalized chordal metric $[z, w]$ on $\mathbb{P}^{1}$. Now the proof of (3.1) is complete.

\section{4. $\mathrm{ON}_{\mathrm{Per}_{c}^{*}}(n), \operatorname{Per}_{c}(n), \operatorname{AND} p_{n}^{*}(\lambda, w)$}

We begin with a notion from the number theory; see, e.g., [1, Chapter 2].

Definition 4.1. The Möbius function $\mu: \mathbb{N} \rightarrow\{0, \pm 1\}$ is defined by $\mu(1)=1$ and, for every $n \geq 2$, by $\mu(n)=0$ if $p^{2} \mid n$ for some prime number $p$, and $\mu(n)=(-1)^{\ell}$ if $n$ factors as a product of distinct $\ell$ prime numbers.

Let $f: \Lambda \times \mathbb{P}^{1} \rightarrow \mathbb{P}^{1}$ be a holomorphic family of rational functions on $\mathbb{P}^{1}$ of degree $d>1$ over a connected complex manifold $\Lambda$.

Definition 4.2 (A periodic point having the formally exact period). Fix $\lambda \in \Lambda$ and $n \in \mathbb{N}$. A fixed point $w \in \mathbb{P}^{1}$ of $f_{\lambda}^{n}$ is a periodic point of $f_{\lambda}$ having the formally exact period $n$ if either

(i) $w \in \operatorname{Fix}^{*}\left(f_{\lambda}^{n}\right)$ or

(ii) there is an $m \in \mathbb{N}$ satisfying $m \mid n$ and $m<n$ such that $w \in \operatorname{Fix}^{*}\left(f_{\lambda}^{m}\right)$ and that $\left(f_{\lambda}^{m}\right)^{\prime}(w)$ is a primitive $(n / m)$-th root of unity.

Let $\operatorname{Fix}^{* *}\left(f_{\lambda}^{n}\right)$ be the set of all periodic points of $f_{\lambda}$ having the formally exact period $n$.

Remark 4.3. For every distinct $n, m \in \mathbb{N}$, $\operatorname{Fix}^{*}\left(f_{\lambda}^{n}\right) \cap \operatorname{Fix}^{*}\left(f_{\lambda}^{m}\right)=\emptyset$, but $\operatorname{Fix}^{* *}\left(f_{\lambda}^{n}\right) \cap$ $\operatorname{Fix}^{* *}\left(f_{\lambda}^{m}\right)$ might be non-empty.

For every $\lambda_{0} \in \Lambda$, choose an open and connected neighborhood $U$ of $\lambda_{0}$ in $\Lambda$ so small that there is a lift $\tilde{f}: U \times \mathbb{C}^{2} \rightarrow \mathbb{C}^{2}$ of $f$, and set $\tilde{f}_{\lambda}=\tilde{f}(\lambda, \cdot)$, as before.

4.1. Fundamental facts. For the proof of the following facts, see e.g. Silverman [28, Theorem 4.5] and Berteloot [6, §2.3.2].

Fact 4.4 (Holomorphic family of dynatomic polynomials). For every $n \in \mathbb{N}$, the function

$$
\Phi_{\tilde{f}, n}^{*}(\lambda, p):=\prod_{m \in \mathbb{N}: m \mid n}\left(\tilde{f}_{\lambda}^{m}(p) \wedge p\right)^{\mu(n / m)} \quad \text { on } U \times \mathbb{C}^{2}
$$

is holomorphic, and for every $\lambda \in U, \Phi_{\tilde{f}, n}^{*}(\lambda, \cdot)$ is a homogeneous polynomial on $\mathbb{C}^{2}$ of degree $\nu(n)=\sum_{m \in \mathbb{N}: m \mid n} \mu(n / m)\left(d^{m}+1\right)$, which is determined by $n$ (and $d$ ) and is independent of $\lambda$. By the Möbius inversion formula (cf. [1, Chapter 2]), (4.1) is equivalent to

$$
\tilde{f}_{\lambda}^{n}(p) \wedge p=\prod_{m \in \mathbb{N}: m \mid n} \Phi_{\tilde{f}, m}^{*}(\lambda, p) \quad \text { on } U \times \mathbb{C}^{2}
$$


Fact 4.5 . For every $n \in \mathbb{N}$ and every $\lambda \in U$, we can choose $\left(\tilde{z}_{k}^{(n)}(\lambda)\right)_{k=1}^{\nu(n)}$ in $\mathbb{C}^{2} \backslash\{0\}$ such that the homogeneous polynomial $\Phi_{\tilde{f}, n}^{*}(\lambda, \cdot)$ factors as

$$
\Phi_{\tilde{f}, n}^{*}(\lambda, p)=\prod_{k=1}^{\nu(n)}\left(p \wedge \tilde{z}_{k}^{(n)}(\lambda)\right) \quad \text { on } \mathbb{C}^{2}
$$

Setting $z_{k}^{(n)}(\lambda):=\pi\left(\tilde{z}_{k}^{(n)}(\lambda)\right)$ for each $k \in\{1,2, \ldots, \nu(n)\}$, we indeed have

$$
\left\{z_{k}^{(n)}(\lambda): k \in\{1, \ldots, \nu(n)\}\right\}=\operatorname{Fix}^{* *}\left(f_{\lambda}\right) .
$$

Moreover, up to its permutation, the sequence $\left(z_{k}^{(n)}(\lambda)\right)_{k=1}^{\nu(n)}$ in $\mathbb{P}^{1}$ is determined by $f, n$ and $\lambda$ and depends on choices of neither $\tilde{f}$ nor $\left(\tilde{z}_{k}^{(n)}(\lambda)\right)_{k=1}^{\nu(n)}$.

4.2. Local description of $\operatorname{Per}_{c}^{*}(n)$ and a global decomposition of $\operatorname{Per}_{c}(n)$. In addition to $\tilde{f}$, for every marked critical point $c: \Lambda \rightarrow \mathbb{P}^{1}$ of $f$, decreasing $U$ if necessary, there is also a lift $\tilde{c}: U \rightarrow \mathbb{C}^{2} \backslash\{0\}$ of $c$. For every $n \in \mathbb{N}$, recall that $\tilde{F}_{n}(\lambda):=\tilde{f}_{\lambda}^{n}(\tilde{c}(\lambda))$ on $U$, and define the function

$$
\tilde{H}_{n}(\lambda)=\tilde{H}_{\tilde{f}, n}^{\tilde{c}}(\lambda):=\Phi_{\tilde{f}, n}^{*}(\lambda, \tilde{c}(\lambda)) \quad \text { on } U,
$$

which is holomorphic by Fact 4.4. Then by (4.3),

$$
\tilde{H}_{n}=\prod_{k=1}^{\nu(n)}\left(\tilde{c} \wedge \tilde{z}_{k}^{(n)}\right) \quad \text { on } U,
$$

and by (4.2),

$$
\tilde{F}_{n} \wedge \tilde{c}=\prod_{m \in \mathbb{N}: m \mid n} \tilde{H}_{m} \text { on } U .
$$

Lemma 4.6 (A local description of $\operatorname{Per}_{c}^{*}(n)$ ). For every $n \in \mathbb{N}$,

$$
\operatorname{Per}_{c}^{*}(n) \mid U=\left[\tilde{H}_{n}=0\right] \text {. }
$$

Proof. For every $n \in \mathbb{N}$, we claim that

$$
X_{n}^{*}:=\operatorname{supp}\left(\operatorname{Per}_{c}^{*}(n) \mid U\right)=\operatorname{supp}\left[\tilde{H}_{n}=0\right] ;
$$

for, we have

$$
\begin{aligned}
\operatorname{supp}\left(\operatorname{Per}_{c}^{*}(n) \mid U\right) & =\left\{\lambda \in U: c(\lambda) \in \operatorname{Fix}^{*}\left(f_{\lambda}^{n}\right)\right\} & (\text { by (1.1) and (1.2)) } \\
& =\left\{\lambda \in U: c(\lambda) \in \operatorname{Fix}^{* *}\left(f_{\lambda}^{n}\right)\right\} & \left(\text { by }\left(f_{\lambda}^{n}\right)^{\prime}(c(\lambda))=0 \neq 1\right) \\
& =\operatorname{supp}\left[\tilde{H}_{n}=0\right] & \text { (by (4.6) and (4.4)). }
\end{aligned}
$$

This also implies that for every distinct $m, n \in \mathbb{N}$,

$$
\begin{aligned}
\operatorname{supp}\left[\tilde{H}_{m}=0\right] \cap \operatorname{supp}\left[\tilde{H}_{n}=0\right] & \\
& =\left\{\lambda \in U: c(\lambda) \in \operatorname{Fix}^{*}\left(f_{\lambda}^{m}\right) \cap \operatorname{Fix}^{*}\left(f_{\lambda}^{n}\right)(=\emptyset)\right\}=\emptyset .
\end{aligned}
$$

Fix $n \in \mathbb{N}$. We claim that for every irreducible component $V$ of $X_{n}^{*}, \operatorname{ord}_{V}\left(\operatorname{Per}_{c}^{*}(n) \mid U\right)$ $=\operatorname{ord}_{V}\left[\tilde{H}_{n}=0\right]$; for, we have

$$
\begin{array}{rlr}
\operatorname{ord}_{V}\left(\operatorname{Per}_{c}^{*}(n) \mid U\right) & =\operatorname{ord}_{V}\left(\operatorname{Per}_{c}(n) \mid U\right) & \\
& =\operatorname{ord}_{V}\left[\tilde{F}_{n} \wedge \tilde{c}=0\right] & \\
& =\operatorname{ord}_{V}\left[\tilde{H}_{n}=0\right] & \text { (by (1.2)) }
\end{array}
$$

Now the proof is complete. 
For every $n \in \mathbb{N}$, recall also that $F_{n}(\lambda):=f_{\lambda}^{n}(c(\lambda))$ on $\Lambda$.

Lemma 4.7 (A global decomposition of $\operatorname{Per}_{c}(n)$ ). For every $n \in \mathbb{N}$, under the assumption that $F_{n} \not \equiv c$ on $\Lambda$, it holds that

$$
\operatorname{Per}_{c}(n)=\sum_{m \in \mathbb{N}: m \mid n} \operatorname{Per}_{c}^{*}(m) \text { on } \Lambda \text {. }
$$

Proof. Fix $n \in \mathbb{N}$. By (2.3), (4.7), and (4.8), if $F_{n} \not \equiv c$ on $U$, then $\operatorname{Per}_{c}(n) \mid U=$ $\sum_{m \in \mathbb{N}: m \mid n} \operatorname{Per}_{c}^{*}(m) \mid U$, so (4.11) holds since $\lambda_{0}$ is arbitrary.

4.3. The definition of $p_{n}^{*}(\lambda, w)$. For the details, see Berteloot [6, §2.3.1].

For every $n \in \mathbb{N}$, every $\lambda \in \Lambda$, and every $j \in\{0,1,2, \ldots, \nu(n)\}$, let $\sigma_{j}^{*}(n, \lambda)$ be the $j$-th elementary symmetric function associated to $\left(\left(f_{\lambda}^{n}\right)^{\prime}\left(z_{k}^{(n)}(\lambda)\right)\right)_{k=1}^{\nu(n)}$.

Then, for every $n \in \mathbb{N}$, by the holomorphy of $\Phi_{\tilde{f}, n}^{*}$ and $f$, the function $\sigma_{j}^{*}(n, \cdot)$ is holomorphic on $\Lambda$ for every $j \in\{0,1,2, \ldots, \nu(n)\}$.

Definition 4.8 (cf. [4, §2.1]). For every $n \in \mathbb{N}$, there is a holomorphic function $p_{n}^{*}(\lambda, w)=p_{f, n}^{*}(\lambda, w)$ on $\Lambda \times \mathbb{C}$, which is unique up to multiplication in $n$-th roots of unity, such that

$$
\left(p_{n}^{*}(\lambda, w)\right)^{n}=\sum_{j=0}^{\nu(n)} \sigma_{j}^{*}(n, \lambda)(-w)^{\nu(n)-j} \quad \text { on } \Lambda \times \mathbb{C} .
$$

For every $n \in \mathbb{N}$ and every $\lambda \in \Lambda$, we have

$$
\left|p_{n}^{*}(\lambda, 0)\right|
$$

$$
=\left|\left(\sigma_{\nu(n)}^{*}(n, \lambda)\right)^{1 / n}\right|=\prod_{k=1}^{\nu(n)}\left|\left(f_{\lambda}^{n}\right)^{\prime}\left(z_{k}^{(n)}(\lambda)\right)\right|^{1 / n}=\prod_{z \in \operatorname{Fix}^{*}\left(f_{\lambda}^{n}\right)}\left|\left(f_{\lambda}^{n}\right)^{\prime}(z)\right|^{1 / n},
$$

where the final equality holds since for every $k \in\{1,2, \ldots, \nu(n)\}$ satisfying

$$
\left|\left(f_{\lambda}^{n}\right)^{\prime}\left(z_{k}^{(n)}(\lambda)\right)\right| \neq 1
$$

$z_{k}^{(n)}(\lambda)$ is in $\operatorname{Fix}^{*}\left(f_{\lambda}^{n}\right)$ and is a simple root of $\Phi_{\tilde{f}, n}^{*}(\lambda, \cdot)$.

Hence, by the chain rule, we have not only (1.7) but also

$$
\left|p_{n}^{*}(\lambda, 0)\right|=\prod_{k=1}^{\nu(n)}\left|f_{\lambda}^{\prime}\left(z_{k}^{(n)}(\lambda)\right)\right|
$$

\section{Proof of Theorem 1}

5.1. Basic facts. Following Bassanelli-Berteloot [4, Theorem 2.5], we refer to the following as a compactness principle for subharmonic functions.

Theorem 5.1 ([16, a consequence of Theorem 4.1.9 (a)]). Let $\left(\phi_{j}\right)$ be a sequence of subharmonic functions on a domain $U$ in $\mathbb{R}^{n}$, and suppose that $\left(\phi_{j}\right)$ is locally uniformly bounded from above. If $\phi:=\lim _{j \rightarrow \infty} \phi_{j}$ exists Lebesgue a.e. on $U$, then indeed $\lim _{j \rightarrow \infty} \phi_{j}=\phi$ in $L_{\mathrm{loc}}^{1}(U)$. 
5.2. Proof of Theorem 1, Let $f$ and $c$ be as in Theorem 1, and recall that $F_{n}(\lambda):=f_{\lambda}^{n}(c(\lambda))$ on $\Lambda$ for each $n \in \mathbb{N}$. By Remark 1.4 we can assume without loss of generality that

$$
F_{n} \not \equiv c \quad \text { on } \Lambda \quad \text { for every } n \in \mathbb{N} \text {. }
$$

For every $\lambda_{0} \in \Lambda$, choose an open and connected neighborhood $U$ of $\lambda_{0}$ in $\Lambda$ so small that there are a lift $\tilde{f}: U \times \mathbb{C}^{2} \rightarrow \mathbb{C}^{2}$ of $f$ and a lift $\tilde{c}: U \rightarrow \mathbb{C}^{2} \backslash\{0\}$ of $c$. Recall that $\tilde{f}_{\lambda}=\tilde{f}(\lambda, \cdot)$, that for every $n \in \mathbb{N}, \tilde{F}_{n}(\lambda):=\tilde{f}_{\lambda}^{n}(\tilde{c}(\lambda))$ on $U$ and $\log \left|\tilde{F}_{n} \wedge \tilde{c}\right|=$ $\log \left[F_{n}, c\right]+\log \left\|\tilde{F}_{n}\right\|+\log \|\tilde{c}\|$ on $U($ cf. (2.4) $)$, and that $\lim _{n \rightarrow \infty}\left(\log \left\|\tilde{F}_{n}(\lambda)\right\|\right) / d^{n}=$ $G^{\lambda}(\tilde{c}(\lambda))$ locally uniformly on $U$ (cf. (2.6) $)$.

Let us first prove (1.4) and then prove (1.5).

Proof of (1.4). According to Lemma 2.2, it is sufficient to prove (1.41). Let us show (1.4I). By (2.6) and (2.4), the sequence $\left(\left(\log \left|\tilde{F}_{n} \wedge \tilde{c}\right|\right) /\left(d^{n}+1\right)\right)$ of plurisubharmonic functions on $U$ is locally uniformly bounded from above on $U$.

Claim.

$$
\lim _{n \rightarrow \infty} \frac{\log \left|\tilde{F}_{n}(\lambda) \wedge \tilde{c}(\lambda)\right|}{d^{n}+1}=G^{\lambda}(\tilde{c}(\lambda)) \quad \text { for Lebesgue a.e. } \lambda \in U
$$

Proof. By the assumption (5.1), the union $\bigcup_{n \in \mathbb{N}} \operatorname{supp}\left[F_{n}=c\right]$ is a Lebesgue null subset in $\Lambda$, and by (2.4), Lemma 3.2, and (2.6), for every $\lambda \in U \backslash \bigcup_{n \in \mathbb{N}} \operatorname{supp}\left[F_{n}=c\right]$,

$$
\begin{aligned}
\lim _{n \rightarrow \infty} \frac{\log \left|\tilde{F}_{n}(\lambda) \wedge \tilde{c}(\lambda)\right|}{d^{n}+1}=\lim _{n \rightarrow \infty} \frac{\log \left[f_{\lambda}^{n}(c(\lambda)), c(\lambda)\right]}{d^{n}+1} & +\lim _{n \rightarrow \infty} \frac{\log \left\|\tilde{F}_{n}(\lambda)\right\|}{d^{n}} \\
& =0+G^{\lambda}(\tilde{c}(\lambda))=G^{\lambda}(\tilde{c}(\lambda)) .
\end{aligned}
$$

This completes the proof.

By this claim and Theorem 5.1 (a compactness principle), using also (2.6) and (2.4), we have

$$
\begin{array}{r}
\lim _{n \rightarrow \infty} \frac{\log \left[F_{n}(\lambda), c(\lambda)\right]}{d^{n}+1}=\lim _{n \rightarrow \infty} \frac{\log \left|\tilde{F}_{n}(\lambda) \wedge \tilde{c}(\lambda)\right|}{d^{n}+1}-\lim _{n \rightarrow \infty} \frac{\log \left\|\tilde{F}_{n}(\lambda)\right\|}{d^{n}} \\
\quad=G^{\lambda}(\tilde{c}(\lambda))-G^{\lambda}(\tilde{c}(\lambda))=0 \quad \text { in } L_{\text {loc }}^{1}(U) .
\end{array}
$$

Since $\lambda_{0}$ is arbitrary, the proof of (1.4]), and so of (1.4), is complete.

Proof of (1.5). Under the assumption (5.1), by the Möbius inversion of the global decomposition (4.11) of $\operatorname{Per}_{c}(n)$ (in Lemma 4.7), for every smooth $\left(\operatorname{dim}_{\mathbb{C}} \Lambda-\right.$ $\left.1, \operatorname{dim}_{\mathbb{C}} \Lambda-1\right)$-form $\phi$ on $\Lambda$,

$$
\begin{aligned}
\mid\left\langle\phi, \operatorname{Per}_{c}^{*}(n)\right. & \left.-\operatorname{Per}_{c}(n)\right\rangle \mid \\
\leq & \sum_{m \in \mathbb{N}: m \mid n \text { and } m<n}\left|\mu\left(\frac{n}{m}\right)\right| \cdot\left|\left\langle\phi, \operatorname{Per}_{c}(m)\right\rangle\right|=O\left(d^{n / 2}\right) \quad \text { as } n \rightarrow \infty,
\end{aligned}
$$

where the final order estimate follows from (1.4) and $m \leq n / 2$ for every $m \in \mathbb{N}$ satisfying $m \mid n$ and $m<n$.

Hence (1.4) implies (1.5). 


\section{Proof of Theorem 1.7}

Let $f: \Lambda \times \mathbb{P}^{1} \rightarrow \mathbb{P}^{1}$ be as in Theorem 1.7. Taking a finitely-sheeted possibly ramified covering of $\Lambda$ if necessary, we assume without loss of generality that there are marked critical points $c_{1}, \ldots, c_{2 d-2}: \Lambda \rightarrow \mathbb{P}^{1}$ of $f$ such that for every $\lambda \in$ $\Lambda, c_{1}(\lambda), \ldots, c_{2 d-2}(\lambda)$ are all the critical points of $f_{\lambda}$, taking into account their multiplicities.

Lemma 6.1 (A local decomposition of $\operatorname{Per}_{f}^{*}(n, 0)$ ). For every $\lambda_{0} \in \Lambda$, there are an open neighborhood $U$ of $\lambda_{0}$ in $\Lambda$ and $N_{0} \in \mathbb{N}$ such that for every $n>N_{0}$,

$$
\operatorname{Per}_{f}^{*}(n, 0)=\sum_{j=1}^{2 d-2} \operatorname{Per}_{c_{j}}^{*}(n) \quad \text { on } U .
$$

By Lemma 6.1 and (1.6), the convergence (1.5) in Theorem 1 implies

$$
\lim _{n \rightarrow \infty} \frac{\operatorname{Per}_{f}^{*}(n, 0)}{d^{n}+1}=\lim _{n \rightarrow \infty} \sum_{j=1}^{2 d-2} \frac{\operatorname{Per}_{c_{j}}^{*}(n)}{d^{n}+1}=\sum_{j=1}^{2 d-2} T_{c_{j}}=T_{f} \quad \text { on } U .
$$

Since $\lambda_{0}$ is arbitrary, the convergence (1.9) in Theorem 1.7 holds.

Remark 6.2. For every $n \in \mathbb{N}$ and every $\lambda \in \Lambda$, set $R^{*}\left(f_{\lambda}^{n}\right):=\left\{w \in \operatorname{Fix}^{*}\left(f_{\lambda}^{n}\right)\right.$ : $\left.\left|\left(f_{\lambda}^{n}\right)^{\prime}(w)\right|>1\right\}$. The original proof of Theorem 1.7 is based on the approximation

$$
L\left(f_{\lambda}\right)=\lim _{n \rightarrow \infty} \frac{1}{n d^{n}} \sum_{z \in R^{*}\left(f_{\lambda}^{n}\right)} \log \left|\left(f_{\lambda}^{n}\right)^{\prime}(z)\right| \quad \text { for each } \lambda \in \Lambda ;
$$

for the details of this formula, see Berteloot-Dupont-Molino [7, Corollary 1.6], and also [5, 25]. The proof of Theorem 1.7 presented here does not rely on this approximation and, moreover, the argument developed in the proof of Lemma 6.1. combined with the proof of Theorem 1 is simpler than the original one.

Proof of Lemma 6.1. Fix $\lambda_{0} \in \Lambda$. Choosing an open and connected neighborhood $U$ of $\lambda_{0}$ in $\Lambda$ small enough, we have a lift $\tilde{f}: U \times \mathbb{C}^{2} \rightarrow \mathbb{C}^{2}$ of $f$ and a lift $\tilde{c}_{j}: U \rightarrow \mathbb{C}^{2} \backslash\{0\}$ of $c_{j}$ for every $j \in\{1,2, \ldots, 2 d-2\}$ normalized so that for every $\lambda \in U$, the Jacobian determinant of $\tilde{f}_{\lambda}=\tilde{f}(\lambda, \cdot)$ factors as

$$
\left(\operatorname{det} D \tilde{f}_{\lambda}\right)(p)=\prod_{j=1}^{2 d-2}\left(p \wedge \tilde{c}_{j}(\lambda)\right) \quad \text { on } \mathbb{C}^{2} .
$$

For each $n \in \mathbb{N}$, recall the definition (4.5) of the function $\tilde{H}_{\tilde{f}, n}^{\tilde{c}_{j}}$ on $U$ and set

$$
\tilde{H}_{n}^{(j)}:=\tilde{H}_{\tilde{f}, n}^{\tilde{c}_{j}} \text { on } U, \text { for each } j \in\{1,2, \ldots, 2 d-2\} .
$$

For each $n \in \mathbb{N}$ and each $\lambda \in U$, recall also the definition of $\left(\tilde{z}_{k}^{(n)}(\lambda)\right)_{k=1}^{\nu(n)}$ in $\mathbb{C}^{2} \backslash\{0\}$ and that $z_{k}^{(n)}(\lambda)=\pi\left(\tilde{z}_{k}^{(n)}(\lambda)\right)$ for each $k \in\{1,2, \ldots, \nu(n)\}$, in Fact 4.5.

Claim. For every $n \in \mathbb{N},\left|p_{n}^{*}(\cdot, 0)\right|=\left|\prod_{j=1}^{2 d-2} \tilde{H}_{n}^{(j)}\right| \cdot e^{r_{n}}$ on $U$.

Here $r_{n}(\lambda):=-\nu(n) \log d+2\left(\sum_{k=1}^{\nu(n)} \log \left\|\tilde{f}_{\lambda}\left(\tilde{z}_{k}^{(n)}(\lambda)\right)\right\|-\sum_{k=1}^{\nu(n)} \log \left\|\tilde{z}_{k}^{(n)}(\lambda)\right\|\right)$ is a pointwise finite function on $U$. 
Proof. For every $\lambda \in U$ and every $n \in \mathbb{N}$, by a computation involving Euler's identity (cf. [17, Theorem 4.3]), for every $k \in\{1,2, \ldots, \nu(n)\}$,

$$
\left|f_{\lambda}^{\prime}\left(z_{k}^{(n)}(\lambda)\right)\right|=\frac{1}{d} \frac{\left\|\tilde{f}_{\lambda}\left(\tilde{z}_{k}^{(n)}(\lambda)\right)\right\|^{2}}{\left.\| \tilde{z}_{k}^{(n)}(\lambda)\right) \|^{2}} \mid\left(\operatorname{det}\left(D \tilde{f}_{\lambda}\right)\left(\tilde{z}_{k}^{(n)}(\lambda)\right) \mid,\right.
$$

so by (1.71), we have $\left|p_{n}^{*}(\lambda, 0)\right|=\left|\prod_{k=1}^{\nu(n)}\left(\operatorname{det} D \tilde{f}_{\lambda}\right)\left(\tilde{z}_{k}^{(n)}(\lambda)\right)\right| \cdot e^{r_{n}(\lambda)}$. Moreover, for every $\lambda \in U$ and every $n \in \mathbb{N}$, by (6.2) and (4.6), we have

$$
\prod_{k=1}^{\nu(n)}\left(\operatorname{det} D \tilde{f}_{\lambda}\right)\left(\tilde{z}_{k}^{(n)}(\lambda)\right)=\prod_{k=1}^{\nu(n)} \prod_{j=1}^{2 d-2}\left(\tilde{z}_{k}^{(n)}(\lambda) \wedge \tilde{c}_{j}(\lambda)\right)=\prod_{j=1}^{2 d-2} \tilde{H}_{n}^{(j)}(\lambda),
$$

which completes the proof.

Under the convention $\min \emptyset=0$, set

$$
N_{0}:=\max _{j \in\{1,2, \ldots, 2 d-2\}}\left(\min \left\{n \in \mathbb{N}: f_{\lambda_{0}}^{n}\left(c_{j}\left(\lambda_{0}\right)\right)=c_{j}\left(\lambda_{0}\right)\right\}\right) \in \mathbb{N} \cup\{0\} .
$$

For every $n>N_{0}$, neither $\tilde{H}_{n}^{(j)}$ for every $j \in\{1,2, \ldots, 2 d-2\}$ nor $p_{n}^{*}(\cdot, 0)$ identically vanish on $U$, and by the claim, $p_{n}^{*}(\cdot, 0) /\left(\prod_{j=1}^{2 d-2} \tilde{H}_{n}^{(j)}\right)$ has neither zeros nor poles on $U$. Hence, for every $n>N_{0}$, by the Poincaré-Lelong formula, we have $\left[p_{n}^{*}(\cdot, 0)=\right.$ $0]=\sum_{j=1}^{2 d-2}\left[\tilde{H}_{n}^{(j)}=0\right]$ on $U$, so by the definition (1.8) of $\operatorname{Per}_{f}^{*}(n, 0)$ and the local description (4.8) of $\operatorname{Per}_{c_{j}}^{*}(n)$ (in Lemma 4.6), we have (6.1). Now the proof of Lemma 6.1 is complete.

\section{ACKNOWLEDGEMENT}

The author thanks the referee for a very careful scrutiny and invaluable comments. This research was partially supported by JSPS Grant-in-Aid for Young Scientists (B), 24740087.

\section{REFERENCES}

[1] Tom M. Apostol, Introduction to analytic number theory, Springer-Verlag, New YorkHeidelberg, 1976. Undergraduate Texts in Mathematics. MR0434929 (55 \#7892)

[2] Giovanni Bassanelli and François Berteloot, Bifurcation currents in holomorphic dynamics on $\mathbb{P}^{k}$, J. Reine Angew. Math. 608 (2007), 201-235, DOI 10.1515/CRELLE.2007.058. MR:2339474(2008g:32052)

[3] Giovanni Bassanelli and François Berteloot, Lyapunov exponents, bifurcation currents and laminations in bifurcation loci, Math. Ann. 345 (2009), no. 1, 1-23, DOI 10.1007/s00208008-0325-1. MR2520048 (2011i:37063)

[4] Giovanni Bassanelli and François Berteloot, Distribution of polynomials with cycles of a given multiplier, Nagoya Math. J. 201 (2011), 23-43. MR2772169(2012h:37098)

[5] François Berteloot, Lyapunov exponent of a rational map and multipliers of repelling cycles, Riv. Math. Univ. Parma (N.S.) 1 (2010), no. 2, 263-269. MR2789444 (2012c:37087)

[6] François Berteloot, Bifurcation currents in holomorphic families of rational maps, Pluripotential theory, Lecture Notes in Math., vol. 2075, Springer, Heidelberg, 2013, pp. 1-93, DOI 10.1007/978-3-642-36421-1_1. MR3089068

[7] François Berteloot, Christophe Dupont, and Laura Molino, Normalization of bundle holomorphic contractions and applications to dynamics (English, with English and French summaries), Ann. Inst. Fourier (Grenoble) $\mathbf{5 8}$ (2008), no. 6, 2137-2168. MR2473632 (2009m:32028)

[8] Bodil Branner and John H. Hubbard, The iteration of cubic polynomials. II. Patterns and parapatterns, Acta Math. 169 (1992), no. 3-4, 229-325, DOI 10.1007/BF02392761. MR.1194004(94d:30044) 
[9] X. Buff and T. Gauthier, Quadratic polynomials, multipliers and equidistribution, Proc. Amer. Math. Soc. (to appear).

[10] Demailly, J.-P. Complex analytic and algebraic geometry, available at http://www-fourier.ujfgrenoble.fr/ ^demailly/manuscripts/agbook.pdf (2012).

[11] Laura DeMarco, Dynamics of rational maps: Lyapunov exponents, bifurcations, and capacity, Math. Ann. 326 (2003), no. 1, 43-73, DOI 10.1007/s00208-002-0404-7. MR.1981611 (2004f:32044)

[12] Tien-Cuong Dinh and Nessim Sibony, Dynamics in several complex variables: endomorphisms of projective spaces and polynomial-like mappings, Holomorphic dynamical systems, Lecture Notes in Math., vol. 1998, Springer, Berlin, 2010, pp. 165-294, DOI 10.1007/978-3-642-131714_4. MR2648690 (2011h:32019)

[13] Romain Dujardin, Bifurcation currents and equidistribution in parameter space, Frontiers in Complex Dynamics: in Celebration of John Milnor's 80th birthday, Princeton University Press (2014), 515-566.

[14] Romain Dujardin and Charles Favre, Distribution of rational maps with a preperiodic critical point, Amer. J. Math. 130 (2008), no. 4, 979-1032, DOI 10.1353/ajm.0.0009. MR2427006 (2009f:32035)

[15] T. Gauthier, Equidistribution towards the bifurcation current I : Mulitpliers and degree $d$ polynomials, ArXiv e-prints (Dec. 2013).

[16] Lars Hörmander, The analysis of linear partial differential operators. I: Distribution theory and Fourier analysis, Grundlehren der Mathematischen Wissenschaften [Fundamental Principles of Mathematical Sciences], vol. 256, Springer-Verlag, Berlin, 1983. MR717035 (85g:35002a)

[17] Mattias Jonsson, Sums of Lyapunov exponents for some polynomial maps of $\mathbf{C}^{2}$, Ergodic Theory Dynam. Systems 18 (1998), no. 3, 613-630, DOI 10.1017/S0143385798108209. MR.1631728 (99d:32033)

[18] Maciej Klimek, Pluripotential theory, London Mathematical Society Monographs. New Series, vol. 6, The Clarendon Press, Oxford University Press, New York, 1991. Oxford Science Publications. MR:1150978(93h:32021)

[19] G. M. Levin, On the theory of iterations of polynomial families in the complex plane (Russian), Teor. Funktsir Funktsional. Anal. i Prilozhen. 51 (1989), 94-106, DOI 10.1007/BF01095412; English transl., J. Soviet Math. 52 (1990), no. 6, 3512-3522. MR:1009151 (90j:30040)

[20] M. Yu. Lyubich, Some typical properties of the dynamics of rational mappings (Russian), Uspekhi Mat. Nauk 38 (1983), no. 5(233), 197-198. MR718838 (85f:58063)

[21] R. Mañé, P. Sad, and D. Sullivan, On the dynamics of rational maps, Ann. Sci. École Norm. Sup. (4) 16 (1983), no. 2, 193-217. MR732343 (85j:58089)

[22] Curtis T. McMullen, Complex dynamics and renormalization, Annals of Mathematics Studies, vol. 135, Princeton University Press, Princeton, NJ, 1994. MR 1312365 (96b:58097)

[23] John Milnor, Dynamics in one complex variable, 3rd ed., Annals of Mathematics Studies, vol. 160, Princeton University Press, Princeton, NJ, 2006. MR2193309 (2006g:37070)

[24] Patrick Morton and Franco Vivaldi, Bifurcations and discriminants for polynomial maps, Nonlinearity 8 (1995), no. 4, 571-584. MR1342504 (96k:11028)

[25] Yûsuke Okuyama, Repelling periodic points and logarithmic equidistribution in nonarchimedean dynamics, Acta Arith. 152 (2012), no. 3, 267-277, DOI 10.4064/aa152-3-3. MR2885787

[26] N.-m. Pham, Lyapunov exponents and bifurcation current for polynomial-like maps, arXiv preprint math/0512557 (2005).

[27] Feliks Przytycki, Lyapunov characteristic exponents are nonnegative, Proc. Amer. Math. Soc. 119 (1993), no. 1, 309-317, DOI 10.2307/2159858. MR1186141 (93k:58193)

[28] Joseph H. Silverman, The arithmetic of dynamical systems, Graduate Texts in Mathematics, vol. 241, Springer, New York, 2007. MR2316407 (2008c:11002) JAPAN

Division of Mathematics, Kyoto Institute of Technology, Sakyo-ku, Kyoto 606-8585

E-mail address: okuyama@it.ac.jp 